\title{
End of Life, Food, and Water: Ethical Standards of Care
}

\author{
Stefano Eleuteri, Arianna Caruso, and Ranjeev C. Pulle
}

\begin{abstract}
End-of-life care constitutes an important situation of extreme nutritional vulnerability for older adults. Feeding decisions in late-stage dementia often provoke moral and ethical questions for family members regarding whether or not to continue hand-feeding or opt for tube-feeding placement. Despite the knowledge that starvation and dehydration do not contribute to patient suffering at the end of life and in fact may contribute to a comfortable passage from life, the ethics of not providing artificial nutrition and hydration (ANH) continue to be hotly debated. However, in the past two decades, voluntary stopping of eating and drinking (VSED) has moved from a palliative option of last resort to being increasingly recognized as a valid means to intentionally hasten death for cognitively intact persons dealing with a serious illness. Across many settings globally, when oral intake is deemed unsafe, decisions to withhold oral feeding and to forgo artificial means of providing nutrition are deemed to be ethically and
\end{abstract}

This chapter is a component of Part II: Specialist Versus Generalist Nutritional Care in Aging. For an explanation of the grouping of chapters in this book, please see Chap. 1: "Overview of Nutrition Care in Geriatrics and Orthogeriatrics."

\footnotetext{
S. Eleuteri $(\square)$

Fragility Fracture Network, Sapienza University of Rome, Rome, Italy

e-mail: stefano.eleuteri@uniroma1.it
}

\author{
A. Caruso \\ Academy of Social and Legal Psychology, Rome, Italy \\ R. C. Pulle \\ Internal Medicine Services, The Prince Charles Hospital, Brisbane, Australia \\ e-mail: ChrysRanjeev.Pulle@ health.qld.gov.au
}

Ó. G. Geirsdóttir, J. J. Bell (eds.), Interdisciplinary Nutritional Management and 
legally sanctioned when the decision is made by a capable patient or their legally recognized substitute decision-maker. Decision-making at the end of life involves knowledge of and consideration of the legal, ethical, cultural, religious, and personal values involved in the issue at hand. This chapter attempted to illustrate the unique complexities when considering nutrition therapy (by oral and artificial means) at the end of life.

\section{Keywords}

End of life $\cdot$ Ethics $\cdot$ Artificial nutrition $\cdot$ Hydration $\cdot$ Voluntary stopping of eating and drinking $\cdot$ Older adults

\subsection{Background}

A nutritionally vulnerable older adult has a reduced physical reserve that limits the ability to mount a vigorous recovery in the face of an acute health threat or stressor. Often this vulnerability contributes to more medical complications, longer hospital stays, and increased likelihood of residential aged care admission and, ultimately, end of life [1].

What does it mean to be vulnerable? In the health context, a background of predisposing factors determines the ability of an individual to respond to stressors or precipitants. A vulnerable older person has a greater accumulation of detrimental predisposing factors (e.g., multiple medical conditions, obesity, and limited social support) relative to protective factors (e.g., muscle mass or adipose stores). When an acute stress arises, such as acute inpatient hospitalization for sepsis or surgery, the individual is unable to mount a vigorous recovery and may never fully return to their prestress baseline [1]. The term "nutritionally vulnerable" evokes the classic image of an elderly community dweller with limited resources and/or medical comorbidities that preclude the consumption of a fully adequate diet. Surely, these individuals should appear on the radar of concern in geriatric nutrition, prior to approaching the end of life. However, a very broad array of dynamic challenges contributes to nutritional vulnerability in today's older adults, well before end of life [1].

\subsection{Factors Associated with Nutritional Vulnerability}

Many factors impact the quality and quantity of dietary intake in communitydwelling older adults (Chap. 4). Older adults are predisposed to nutrient deficiency due to a decline in total and resting energy requirements associated with physical inactivity, loss of lean muscle mass, and increased adiposity that gradually reduces food intake while vitamin and mineral needs remain unchanged or increased [2]. Furthermore, biomedical, psychosocial, and environmental and economic factors also seriously impact nutritional status in older adults. In the physiologic sense, vulnerability equates closely with a more familiar term used to describe age-related 
decline, namely, "frailty" (Chap. 8). Frailty is an aging-related clinical syndrome of physiological decline characterized by marked vulnerability to adverse health outcomes increased risk for adverse outcomes including acute illness, disability, falls, hospitalization, need for long-term care, and death [3]. Psychosocial factors that contribute to decreased food intake include depression, social isolation, and loneliness, to name a few. Depression is a prevalent condition in the older population where $7 \%$ have major depressive disorders and up to $17 \%$ have clinically significant depressive symptoms [4]. Risk factors for late-life depression include female gender, lower educational status, loss of a partner, cognitive decline, chronic health conditions, and decline in physical function [5]. The relationship between malnutrition and depression is multifaceted and complex.

Social isolation (objective measure) and loneliness (subjective measure) are common among community-dwelling older adults who live alone, have functional impairments, lack transportation, have low morale, and report limited social networks [6]. Furthermore, social isolation and loneliness are associated with chronic health conditions, cognitive impairment, poor self-reported health, and sleep disorders [7]. Older adults who experience these psychosocial determinants are more likely to eat alone and often have chronically marginal nutrient intake, putting them at a greater risk for malnutrition [8]. Of particular concern are widowed or single older men. These individuals typically have fewer close relationships outside of their spouse, lack cooking skills, or are physically unable to prepare food [9].

In many individuals, such factors associated with nutritional vulnerability will become more focused toward the end of life; this demands the attention of diverse healthcare providers to ensure ethical standards of care are met.

\subsection{Malnutrition: A Commonly Identified Precursor to the End of Life}

Food insecurity, defined as "limited or uncertain availability of nutritionally adequate and safe foods or limited or uncertain ability to acquire acceptable foods in socially acceptable ways," encompasses not only the lack of economic resources to obtain nutritionally adequate food but also the inability to access and appropriately use food. According to the USDA, in 2013, the prevalence of food insecurity in the United States was $14.3 \%$ of households. Among older adults, $8.7 \%$ of households with an older adult and $9.0 \%$ of older adults were living alone with insecure food [10].

The multidimensional phenomenon of food insecurity is peculiar in this population $[11,12]$. In older populations, food insecurity results from more than financial resource constraints. Functional impairment, not owning a home, isolation, gender, financial vulnerability, and poor health have statistically significant associations with food insecurity. These associations suggest that differences in food use between older and younger populations should be considered. These important risk factors for food insecurity tend to occur together, which results in a much higher risk for food insecurity in older populations; this again is likely to be a focal point toward the end of life $[11,13,14]$. 
Shortcomings in past approaches used to assess nutritional status have hindered efforts to describe the extent and degree of malnutrition and nutritional risk for older adults in clinical settings. The use of assessment tools like the Mini Nutritional Assessment (MNA) is not routinely applied across the clinical environment, and serum albumin level, although it is heavily relied upon as a sole indicator of nutritional status, actually reflects primarily the inflammatory state rather than the true body store of essential nutrients [15] (Chap. 3). Fortunately, as detailed in Chap. 3, new paradigms describing malnutrition have been recently developed and codified [16-18]. These approaches more accurately reflect the close interrelationship between diseases, substantially improve the accuracy of malnutrition diagnosis, and are anticipated to lead to a better understanding of ways to surmount challenges to nutritional status going forward [1].

Long recognized as a general concern, hospital malnutrition has received considerably more scientific attention in recent years, as evidenced by a number of recent studies and editorials $[19,20]$. As of $2013,14.1 \%$ of the population in the United States was over the age of 65 years, yet this relatively small cohort accounted for the greatest utilization of healthcare services. Older adults made up 34.9\% of all hospital admissions, with a greater mean length of stay and cost per stay than any other age-group [21]. This is because older adults have a higher number of predisposing conditions with comorbid illnesses and disabilities. Malnutrition is a key comorbid illness now recognized as a predictor of adverse events during their hospitalization, such as falls, pressure injuries, unfavorable discharge destination, unplanned readmissions, mortality, and increased healthcare costs [15, 22-25]. While it is clear that people approaching end of life are high users of healthcare, ongoing works are required to understand if variations in hospitalization and healthcare costs may be attributable to variation in the quantity or quality of end-of-life care available, for example, ensuring processes to manage nutritional vulnerability [26, 27].

This combination of susceptibility and reduced ability to overcome stressors leads to poor long-term outcomes and commonly results in loss of independence, which is a marker of frailty (Chap. 8). The decline of all these physiological parameters can, in many cases, be directly related to reduced energy intake. When energy intake is insufficient to meet the demands of the body either due to starvation, acute illness, or chronic disease/disability, then malnutrition becomes the driver that leads to the further deterioration of functional ability and inability to recover from disease [1] (Chaps. 2-5).

Several recent publications [28, 29] addressing nutritional risk in older patients found that malnutrition in hospitalized geriatric patients is associated with an increased risk of death at 3 months. These papers have further illuminated the profile of malnutrition to include more clinical characteristics, including eating difficulties (Chap. 18) and other functional limitations, depression, polypharmacy (Chap. 20), pressure ulcers (Chap. 15), and cognitive impairment, as well as social factors and hospital factors such as unnecessarily lengthy periods of medical orders requiring patients to have nothing by mouth (NPO), frequent meal interruptions, and low acceptance of foods served (Chaps. 1-6) [29]. It is imperative to recognize both malnutrition and risk of malnutrition as early as possible during the hospital admission process so that all of the medical, psychological, and functional factors 
related to malnutrition can be addressed by an interprofessional team focusing on the patient's preferences regarding food choices, timing of meals, and/or selffeeding strategies within their individual social and environmental circumstances [1].

\subsubsection{Nutrition at the End of Life}

The extreme nutritional vulnerability for older adults is often most acutely observed toward end-of-life care. As an example, feeding decisions in advanced cancer or late-stage dementia often provoke wrenching moral and ethical questions for family members regarding whether or not to continue dedicated hand-feeding and nutritional supplementation or even to consider tube-feeding placement, noting that in many situations, these may be unlikely to prolong life and improve quality of life or may be considered medically futile $[1,30]$.

From the outset, we consequently note that nutritional intervention in this phase of life should be individualized [30], particularly given there are no clear criteria to ascertain the beginning of the dying phase.

In patients at the end of life (survival days or weeks), artificial hydration and nutrition pose clinical, ethical, and logistical dilemmas resulting in debates for and against such interventions [30]. No strong evidence exists supporting the use of "artificial" tube feeding, for example, parenteral or enteral tube feeding for hydration and nutrition for terminally ill patients; however, a paucity of research examining the issue exists [30-32]. Patients during the last days and weeks of life often have anorexia, decreased oral intake, resulting in sarcopenia and obvious frailty, which phenotypically present as loss of body weight with reduced muscle mass and adipose tissue. In addition, cancer patients, frequently gastrointestinal or patients with metastatic malignancy, may develop mechanical obstruction of the digestive tract preventing enteral nutrition [33]. While these may appear confronting to carers and loved ones looking on, in these patients, the goals of therapy should routinely be directed at symptomatic management rather than reversing nutritional deficits. For most, the pleasure of tasting food and the social benefits of participating in meals with family and friends should be emphasized over increasing caloric intake [31]. When patients approach end of life, they often have severely restricted oral intake of food and fluids. The overwhelming principles toward end-of-life nutritional care should revolve not only around a patient's pre-existing wishes regarding intervention but also on comfort measures [32]. However, this should not automatically preclude consideration of artificial nutrition and hydration for individual older adults at the end of life.

\subsection{Artificial Nutrition and Hydration (ANH)}

Despite the knowledge that starvation and dehydration do not contribute to patient suffering at the end of life and in fact may contribute to a comfortable passage from life, the ethics of providing or not providing artificial nutrition and hydration (ANH) continue to be hotly debated [34]. ANH are defined as a group of medical treatments 
provided to patients who cannot meet their daily requirements orally, with resultant malnutrition, electrolyte abnormalities, and/or metabolic derangements. Various modalities to deliver ANH include intravenous hydration and intravenous parenteral nutrition, nasogastric feeding, and placement of surgical feeding devices to deliver the required hydration and nourishment.

In patients at the end of life (survival days or weeks), nutrition support and particularly artificial hydration pose clinical, ethical, and logistical dilemmas resulting in debates for and against such interventions [30]. While there has been a lack of strong evidence supporting the use of "artificial" tube feeding, for example, parenteral or enteral tube feeding for hydration and nutrition for terminally ill patients [30-32], we note that artificial nutrition has become an accepted part of palliative care for at least some situations [32]. Being medical treatments, the initiation, termination, and withholding of these modalities must be medically and ethically justified $[32,35]$. Many investigators have found that dying persons overwhelmingly deny thirst and hunger; and application of lubricants or provision of ice chips and oral hygiene alleviates many xerostomic complaints. Provision of oral food and fluids should be strongly encouraged, as in most situations, they give comfort, pleasure, a sense of autonomy, and dignity and are viewed as essential caregiving [34].

The practice of medical nutrition and hydration provokes both supportive and opposing views. Some ethicists would argue that the symptom of thirst should be addressed, because, without it, the patient will experience confusion, restlessness, or neuromuscular weakness, thus decreasing the patient's quality of life. Others may argue that the terminally ill patient with declining renal function receiving artificial hydration will suffer from choking on increased secretions, pulmonary edema, and ascites [36]. Alternatively, while offering an unrestricted diet for patients with dysphagia may increase the risk of aspiration or choking, this may also provide a basic comfort and potentially final right of life in end-stage disease. In order to provide artificial nutrition and hydration, medical devices such as urinary catheters and surgically placed feeding tubes may be required; whether these add to or subtract from quality of life should be considered on an individual basis. For example, a patient with decreased mental capacity may try to remove these devices. Attempting to continue the therapy, caregivers may use restraints or sedation, resulting in an inappropriate decrease in quality of life [37].

Families may believe that hydration decreases pain, replenishes the body, enhances effectiveness of medications, and in general can make the patient feel better both mentally and physically. At the end of life, families may feel that they are responsible for maintaining their loved one's dignity, and continued hydration may contribute to their perception that this is being accomplished. In some cases, the family's insistence that the patient take nutrition may cause conflict even before the implementation of ANH becomes the only option. Strong beliefs in the value of nutrition and hydration at the end of life may give the family some satisfaction that they are helping the patient [37]. The patient's refusal to eat may exacerbate the family's feeling of helplessness. Families' perceptions of the importance of nutrition may also color their impression of the healthcare team dedication. If the family believes that the medical team is not placing enough importance on nutrition and hydration, this may translate into the perception that the healthcare team is 
negligent [37]. This is where good communication between clinical team and families is required to discuss goals and directions of care (Chaps. 10, 11, and 13).

Patients at the end of life lose interest in eating and have fatigue, altered body image, and decreased ability to digest. These are all highly correlated with psychological distress [38]. Patients may believe that if they are not able to take food and fluid orally, then ANH will help them survive by preventing dehydration and increasing physical strength. They may also believe that a gastrostomy or a nasogastric tube can make their quality of life worse. If it is not something that will cure their illness, patients may decline ANH [36]. Patients may believe that ANH is a symbol of their families' love for them, an important part of their meticulous care for their health and well-being [39].

Some patients and families choose to implement ANH rather than go without, while some others do not wish to be tube-fed [33, 40, 41]. It is important to stress that, to be considered medically ethical and justified, ANH must provide a benefit to the patient (beneficence), avoid harm (non-maleficence), be in accordance with the patient's wishes (autonomy), and avoid overutilization of resource for one patient with harm to others and be available to all patients in similar circumstances (justice) [32, 37]. The possible benefits of feeding via ANH include improvement in the patient's quality of life and improved nutrition with decreased incidence of bedsores and other infections [35].

However, these treatment modalities are not without risk to the patient. ANH can lead to fluid overload and electrolyte/metabolic derangements, aspiration pneumonitis, or pneumonia and are associated with an increased risk of infection [42]. Moreover, the procedures involved with feeding device placement can themselves lead to increased morbidity and mortality [43]. Finally, while there is no evidence that withholding nourishment and hydration in terminal illness causes pain or suffering, some clinicians would argue that it does not prolong life but only prolongs the dying process [35]. It is the balance between benefits of ANH and potential disadvantages that the clinician needs to fully convey to patient and family before embarking on this course of intervention.

Arguably, in many cases, the decision to offer artificial feeding or not revolves less around the benefits versus risks of the intervention but whether or not terminally ill patients and their family have emotionally accepted the fact the patient is dying [31]. We conclude this section by noting that the decision to administer parenteral or enteral tube feeding should be individualized, based upon the clinical scenario, and should also be consistent with the goals of care of the patient $[41,44]$. In case of uncertainty of the benefits and risks of artificial feeding in a particular patient, a brief trial with clearly defined goals may be appropriate to initiate, followed by reassessments of its clinical benefits and harm.

\subsection{Voluntary Stopping Eating and Drinking (VSED)}

In the past two decades, VSED has moved from a palliative option of last resort [45] to being increasingly recognized as a valid means to intentionally hasten death for cognitively intact persons dealing with a serious illness [32, 46, 47]. It should be 
noted that VSED is different from declining interest for food and water at the end of life [48]. Properly defined, VSED refers to "a conscious and deliberate decision, by a capacitated patient suffering from advanced illness or an extremely debilitating medical condition, to intentionally refrain from receiving food or fluids by mouth, to hasten death" [49]. Those who have cared for persons choosing VSED have noted the following patient motivations for undertaking this method: willingness to die, feeling of senseless in continued life, experiencing poor quality of life, and desire to control the dying process [50]. Though considerable legal and ethical analysis has been undertaken over the past decade to clarify the moral and legal standing of VSED, continued controversy and uncertainty around its use center on the following concerns: Is it legal to offer standard palliative care to patients undertaking VSED without being viewed as "assisting" in their suicide? If it is legal to support VSED, can a practitioner exercise conscientious objection and refuse to support a patient requesting it? Would providing advice to a patient about VSED as an option potentially be construed as "encouraging suicide" [48]? If a patient and family choose VSED, is it a reflection that the patient has entered a terminal or palliative state? These are all questions that should be asked and considered in relation to local contexts and the will of the patient and their informed consent [32]. We conclude this section by noting the ESPEN guideline on ethical aspects of artificial nutrition and hydration Statements 14 "the renouncement of food and drink may be regarded as an expression of self-determined dying by way of an autonomous decision towards one's own life, but should not be confused with severe depression or disease related lack of appetite," and 34 "Voluntary cessation of nutrition and hydration is a legally and medically acceptable decision of a competent patient, when chosen in disease conditions with frustrating prognosis and at the end life."

\subsection{Stopping Oral Nutrition for Incapable Patients}

When oral intake is deemed unsafe, decisions to withhold oral feeding and to forgo artificial means of providing nutrition are deemed to be ethically and legally sanctioned when the decision is made by a capable patient or their legally recognized substitute decision-maker. The issue arises when the family attempts to withhold nutrition from incapable patients even if feeding is safe and accepted by patients. The motivation behind families requesting staff to stop oral hydration or nutrition is often based on an interpretation of a prior expressed wish. However, in many cases, these verbal or written instructions make no explicit reference to hand-feeding. Families may interpret assisted hand-feeding as a life-prolonging intervention; however, for the healthcare team, supporting the family's request is interpreted as condoning neglect and endangerment of a vulnerable patient [51]. This is where the goals of care need to be addressed between clinical team and patient. The choice of withholding or ceasing nutrition care in these patients is a difficult task that should be carefully discussed with caregivers; even then, emotional and/or ethical conflicts among family, carers, and/or healthcare team members will undoubtedly arise from time to time [32]. 


\subsection{Conclusions and Take-Home Points}

Decision-making at the end of life involves knowledge and consideration of the legal, ethical, cultural, religious, and personal values involved in the issue at hand. This chapter illustrates the unique complexities when considering nutrition therapy (by oral and artificial means) at the end of life. These implications become more poignant in the current world where the aim is comfort at the end of life [52]. Clear goals of care need to be addressed when patient is entering a palliative or terminal phase in their life.

\section{References}

1. Porter Starr KN, McDonald SR, Bales CW (2015) Nutritional vulnerability in older adults: a continuum of concerns. Curr Nutr Rep 4(2):176-184

2. Bernstein M, Munoz N (2012) Position of the Academy of Nutrition and Dietetics: food and nutrition for older adults: promoting health and wellness. J Acad Nutr Diet 112(8):1255-1277

3. Gielen E et al (2012) Musculoskeletal frailty: a geriatric syndrome at the core of fracture occurrence in older age. Calcif Tissue Int 91(3):161-177

4. Luppa $\mathrm{M}$ et al (2012) Age- and gender-specific prevalence of depression in latest-life--systematic review and meta-analysis. J Affect Disord 136(3):212-221

5. Djernes JK (2006) Prevalence and predictors of depression in populations of elderly: a review. Acta Psychiatr Scand 113(5):372-387

6. Coyle CE, Dugan E (2012) Social isolation, loneliness and health among older adults. J Aging Health 24(8):1346-1363

7. Steptoe A et al (2013) Social isolation, loneliness, and all-cause mortality in older men and women. Proc Natl Acad Sci U S A 110(15):5797-5801

8. Romero-Ortuno R et al (2011) Psychosocial and functional correlates of nutrition among community-dwelling older adults in Ireland. J Nutr Health Aging 15(7):527-531

9. Holwerda TJ et al (2012) Increased risk of mortality associated with social isolation in older men: only when feeling lonely? Results from the Amsterdam Study of the Elderly (AMSTEL). Psychol Med 42(4):843-853

10. Alisha Coleman-Jensen CG, Singh A (2014) Household Food Security in the United States in 2013, in Economic Research Report Number 173. United States Department of Agriculture (USDA)

11. Lee JS et al (2011) Food security of older adults requesting older Americans Act Nutrition Program in Georgia can be validly measured using a short form of the U.S. Household Food Security Survey Module. J Nutr 141(7):1362-1368

12. Lee JS, Frongillo EA Jr (2001) Factors associated with food insecurity among U.S. elderly persons: importance of functional impairments. J Gerontol B Psychol Sci Soc Sci 56(2):S94-S99

13. Lee JS, Frongillo EA Jr (2001) Nutritional and health consequences are associated with food insecurity among U.S. elderly persons. J Nutr 131(5):1503-1509

14. Vilar-Compte M et al (2016) Functional limitations, depression, and cash assistance are associated with food insecurity among older urban adults in Mexico City. J Health Care Poor Underserved 27(3): 1537-1554

15. Jensen GL (2006) Inflammation as the key interface of the medical and nutrition universes: a provocative examination of the future of clinical nutrition and medicine. JPEN J Parenter Enteral Nutr 30(5):453-463

16. Jensen GL et al (2019) GLIM criteria for the diagnosis of malnutrition: a consensus report from the global clinical nutrition community. JPEN J Parenter Enteral Nutr 43(1):32-40

17. White JV et al (2012) Consensus statement: Academy of Nutrition and Dietetics and American Society for Parenteral and Enteral Nutrition. J Parenter Enter Nutr 36(3):275-283 
18. Cederholm T et al (2015) Diagnostic criteria for malnutrition - an ESPEN consensus statement. Clin Nutr 34(3):335-340

19. Tappenden KA et al (2013) Critical role of nutrition in improving quality of care: an interdisciplinary call to action to address adult hospital malnutrition. JPEN J Parenter Enteral Nutr 37(4):482-497

20. Corkins MR et al (2014) Malnutrition diagnoses in hospitalized patients: United States, 2010. JPEN J Parenter Enteral Nutr 38(2):186-195

21. Weiss AJ, Elixhauser A (2014) Overview of hospital stays in the United States, 2012: statistical brief \#180. In Healthcare Cost and Utilization Project (HCUP) Statistical Briefs [Internet]. Agency for Healthcare Research and Quality (US), Rockville

22. Fernandez HM et al (2008) House staff member awareness of older inpatients' risks for hazards of hospitalization. Arch Intern Med 168(4):390-396

23. Bell JJ et al (2016) Impact of malnutrition on 12-month mortality following acute hip fracture. ANZ J Surg 86(3):157-161

24. Lackoff AS et al (2020) The association of malnutrition with falls and harm from falls in hospital inpatients: findings from a 5-year observational study. J Clin Nurs 29(3-4):429-436

25. Ness SJ et al (2018) The pressures of obesity: the relationship between obesity, malnutrition and pressure injuries in hospital inpatients. Clin Nutr 37(5):1569-1574

26. Diernberger K et al (2021) Healthcare use and costs in the last year of life: a national population data linkage study. BMJ Support Palliat Care. bmjspcare-2020-002708

27. French EB, McCauley J, Aragon M, Bakx P, Chalkley M, Chen SH, Christensen BJ, Chuang H, Côté-Sergent A, De Nardi M, Fan E, Échevin D, Geoffard PY, Gastaldi-Ménager C, Gørtz M, Ibuka Y, Jones JB, Kallestrup-Lamb M, Karlsson M, Klein TJ, de Lagasnerie G, Michaud PC, O’Donnell O, Rice N, Skinner JS, van Doorslaer E, Ziebarth NR, Kelly E (2017) End-OfLife Medical Spending In Last Twelve Months Of Life Is Lower Than Previously Reported. Health Aff (Millwood). Jul 1;36(7):1211-1217. https://doi.org/10.1377/hlthaff.2017.0174. PMID: 28679807. https://pubmed.ncbi.nlm.nih.gov/28679807/

28. Cerri AP et al (2015) Sarcopenia and malnutrition in acutely ill hospitalized elderly: prevalence and outcomes. Clin Nutr 34(4):745-751

29. Heersink JT et al (2010) Undernutrition in hospitalized older adults: patterns and correlates, outcomes, and opportunities for intervention with a focus on processes of care. J Nutr Elder 29(1):4-41

30. Volkert D et al (2019) ESPEN guideline on clinical nutrition and hydration in geriatrics. Clin Nutr 38(1):10-47

31. Dev R, Dalal S, Bruera E (2012) Is there a role for parenteral nutrition or hydration at the end of life? Curr Opin Support Palliat Care 6(3):365-370

32. Druml C et al (2016) ESPEN guideline on ethical aspects of artificial nutrition and hydration. Clin Nutr 35(3):545-556

33. Duerksen DR et al (2004) Is there a role for TPN in terminally ill patients with bowel obstruction? Nutrition 20(9):760-763

34. Heuberger RA (2010) Artificial nutrition and hydration at the end of life. J Nutr Elder 29(4):347-385

35. van de Vathorst S (2014) Artificial nutrition at the end of life: ethical issues. Best Pract Res Clin Gastroenterol 28(2):247-253

36. Casarett D, Kapo J, Caplan A (2005) Appropriate use of artificial nutrition and hydration-fundamental principles and recommendations. N Engl J Med 353(24):2607-2612

37. Marcolini EG, Putnam AT, Aydin A (2018) History and perspectives on nutrition and hydration at the end of life. Yale J Biol Med 91(2):173-176

38. Del Río MI et al (2012) Hydration and nutrition at the end of life: a systematic review of emotional impact, perceptions, and decision-making among patients, family, and health care staff. Psychooncology 21(9):913-921

39. Chiu TY et al (2004) Terminal cancer patients' wishes and influencing factors toward the provision of artificial nutrition and hydration in Taiwan. J Pain Symptom Manag 27(3):206-214

40. Cohen MZ et al (2012) The meaning of parenteral hydration to family caregivers and patients with advanced cancer receiving hospice care. J Pain Symptom Manag 43(5):855-865 
41. King PC et al (2019) "I wouldn't ever want it": a qualitative evaluation of patient and caregiver perceptions toward enteral tube feeding in hip fracture inpatients. JPEN J Parenter Enteral Nutr 43(4):526-533

42. Jones BJ (2010) Ethics and artificial nutrition towards the end of life. Clin Med (Lond) 10(6):607-610

43. Rahnemai-Azar AA et al (2014) Percutaneous endoscopic gastrostomy: indications, technique, complications and management. World J Gastroenterol 20(24):7739-7751

44. Mon AS, Pulle C, Bell J (2018) Development of an 'enteral tube feeding decision support tool' for hip fracture patients: a modified Delphi approach. Australas J Ageing 37(3):217-223

45. Quill TE, Lo B, Brock DW (1997) Palliative options of last resort: a comparison of voluntarily stopping eating and drinking, terminal sedation, physician-assisted suicide, and voluntary active euthanasia. JAMA 278(23):2099-2104

46. Quill TE et al (2018) Voluntarily stopping eating and drinking among patients with serious advanced illness-clinical, ethical, and legal aspects. JAMA Intern Med 178(1):123-127

47. Jox RJ et al (2017) Voluntary stopping of eating and drinking: is medical support ethically justified? BMC Med 15(1):186

48. McGee A, Miller FG (2017) Advice and care for patients who die by voluntarily stopping eating and drinking is not assisted suicide. BMC Med 15(1):222

49. Pope TM, West A (2014) Legal briefing: voluntarily stopping eating and drinking. J Clin Ethics 25(1):68-80

50. Saladin N, Schnepp W, Fringer A (2018) Voluntary stopping of eating and drinking (VSED) as an unknown challenge in a long-term care institution: an embedded single case study. BMC Nurs 17:39

51. Meier CA, Ong TD (2015) To feed or not to feed? A case report and ethical analysis of withholding food and drink in a patient with advanced dementia. J Pain Symptom Manag 50(6):887-890

52. Henry B (2020) End of life feeding: ethical and legal considerations. Physiol Behav 217:112800

\section{Recommended Reading}

Blair H (2020) End of life feeding: ethical and legal considerations. Physiol Behav 217:112800

Druml C, Ballmer PE, Druml W, Oehmichen F, Shenkin A, Singer P, Soeters P, Weimann A, Bischoff SC (2016) ESPEN guideline on ethical aspects of artificial nutrition and hydration. Clin Nutr 35(3):545-556. https://doi.org/10.1016/j.clnu.2016.02.006. Epub 2016 Feb 16. PMID: 26923519. https://pubmed.ncbi.nlm.nih.gov/26923519/

Quill TE, Ganzini L, Truog RD, Pope TM (2018) Voluntarily stopping eating and drinking among patients with serious advanced illness — clinical, ethical, and legal aspects. JAMA Intern. Med 178(1):123-127

Open Access This chapter is licensed under the terms of the Creative Commons Attribution 4.0 International License (http://creativecommons.org/licenses/by/4.0/), which permits use, sharing, adaptation, distribution and reproduction in any medium or format, as long as you give appropriate credit to the original author(s) and the source, provide a link to the Creative Commons license and indicate if changes were made.

The images or other third party material in this chapter are included in the chapter's Creative Commons license, unless indicated otherwise in a credit line to the material. If material is not included in the chapter's Creative Commons license and your intended use is not permitted by statutory regulation or exceeds the permitted use, you will need to obtain permission directly from the copyright holder.

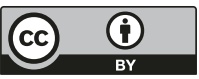

